\title{
Measurements of open heavy-flavour production in pp collisions with ALICE at the LHC
}

\author{
Grazia Luparello* for the ALICE Collaboration \\ Università di Trieste and INFN Trieste \\ E-mail: gluparel@cern.ch
}

\begin{abstract}
In this contribution, an overview of the recent ALICE results on open heavy-flavour production in pp collisions will be reported. Open heavy-flavour production cross sections were measured by ALICE at $\sqrt{s}=7 \mathrm{TeV}$ and $\sqrt{s}=2.76 \mathrm{TeV}$ in a wide $p_{\mathrm{T}}$ interval. The measurements were compared with different perturbative QCD calculations and a good agreement between data and theory was observed.

In addition to heavy-flavour production cross sections, more differential measurements of charm and beauty production are attracting growing interest since they provide further information about the particle production mechanisms. In this perspective, the dependence of heavy-flavour production on charged-particle multiplicity in pp collisions and the azimuthal correlations of heavyflavour hadrons with charged hadrons in pp collisions will be discussed.
\end{abstract}

The European Physical Society Conference on High Energy Physics 22-29 July 2015

Vienna, Austria

*Speaker. 


\section{Introduction}

Heavy quarks, i.e. charm and beauty, are produced in hard partonic scatterings in the initial stages of the hadronic collisions. Therefore, the study of their production in pp collisions at LHC energies provides a test of perturbative QCD (pQCD) calculations at the highest collision energies available. It was observed that theoretical calculations based on pQCD [1, 2, 3] can describe the heavy-flavour $p_{\mathrm{T}}$-dependent cross sections at different collision energies.

More differential measurements of charm and beauty production in pp collisions can provide further information about particle production mechanisms. In particular, heavy-flavour production as a function of charged-particle multiplicity is expected to be sensitive to the interplay between hard and soft QCD processes and could give insight into the role of Multi-Parton Interactions (MPI), i.e. several hard partonic interactions occurring in a single collision between the two protons. Recent results suggest that particle production in pp collisions at LHC energies has a substantial contribution from MPI $[4,5]$. Indications of the relevance of MPI in heavy-flavour production were observed by NA27 [6] and recently by ALICE [7, 8], LHCb [9, 10], CMS [11] and D0 [12]. The measurement of azimuthal correlations between heavy-flavour hadrons and charged particles in pp collisions provides information on the fragmentation and hadronization of heavy quarks. In addition, this study allows us to investigate the different heavy-quark production mechanisms since the various $\mathrm{pQCD}$ processes for the production of a heavy-quark pair influence the azimuthal correlation pattern.

The performance of the ALICE detector allows open heavy-flavour measurements in several decay channels and in a wide rapidity range. At mid-rapidity, open heavy-flavour production is studied by means of fully recontructed D mesons and in the semi-electronic decay channel, while the measurement of non-prompt $\mathrm{J} / \psi$ allows to access the beauty-hadron production cross sections. At forward rapidity, open heavy-flavour production is studied in the semi-muonic decay channel.

\section{Open heavy-flavour $p_{\mathrm{T}}$-differential cross sections}

Cross sections of prompt $\mathrm{D}$ mesons, electrons and muons from decays of heavy-flavour particles and non-prompt $\mathrm{J} / \psi$ were measured by ALICE in pp collisions at both $\sqrt{s}=7 \mathrm{TeV}[13,14$, $15,16,17]$ and $\sqrt{s}=2.76 \mathrm{TeV}[18]$. Different theoretical calculations describe the $p_{\mathrm{T}}$-differential production cross sections within the uncertainties: namely, fixed-order $\mathrm{pQCD}$ calculations relying on the collinear factorisation approach, like GM-VFNS [1] and FONLL [2], or based on the $k_{\mathrm{T}}$-factorisation approach [3]. As an example, Fig. 1 shows the $p_{\mathrm{T}}$-differential cross section at $\sqrt{s}=7 \mathrm{TeV}$ of prompt $\mathrm{D}^{*+}$ mesons (left) and of electrons from charm and beauty hadron decays separately (right) compared with theoretical expectations.

\section{Open heavy-flavour production as a function of charged-particle multiplicity}

The study of heavy-flavour production in pp collisions at $\sqrt{s}=7 \mathrm{TeV}$ as a function of the charged-particle multiplicity is presented via the D-meson self-normalised yield, i.e. the corrected 

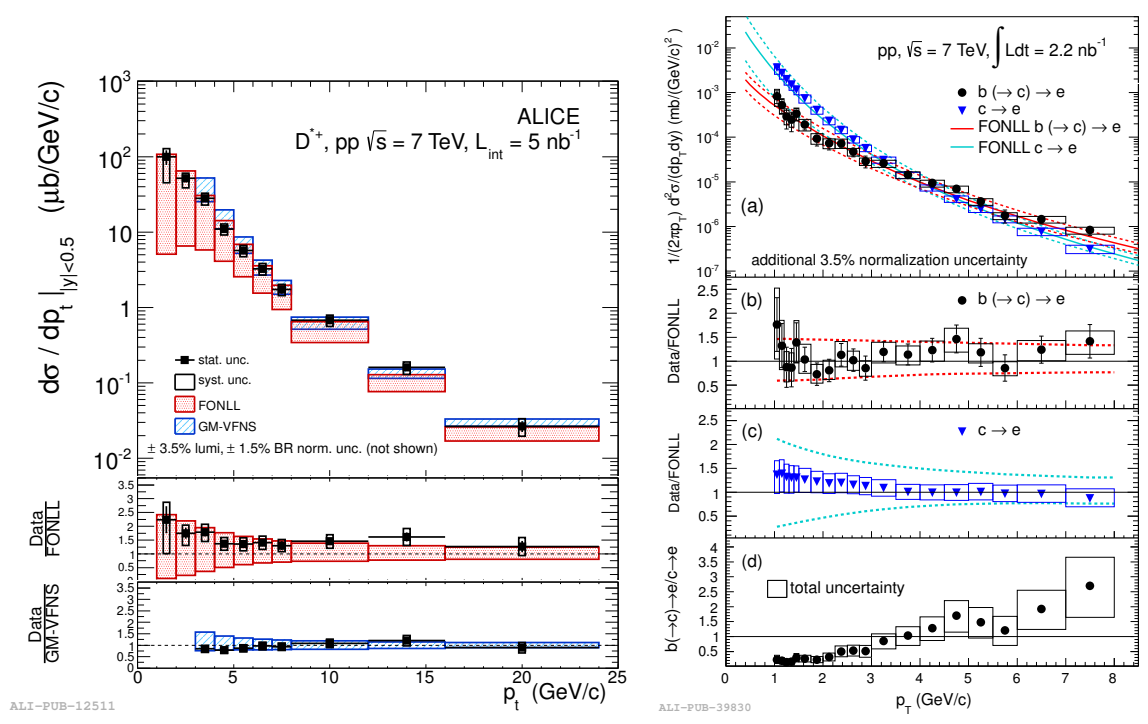

Figure 1: $p_{\mathrm{T}}$-differential cross section of prompt $\mathrm{D}^{*+}$ mesons [13] (left) and of electrons from charm and beauty hadron decays [15] (right).

per-event yield in a given multiplicity interval normalised to the multiplicity-integrated value

$$
\frac{\left(\mathrm{d}^{2} N^{\mathrm{D}} / \mathrm{d} y \mathrm{~d} p_{\mathrm{T}}\right)^{j}}{\left\langle\mathrm{~d}^{2} N^{\mathrm{D}} / \mathrm{d} y \mathrm{~d} p_{\mathrm{T}}\right\rangle}=\left(\frac{1}{N_{\text {events }}^{j}} \frac{N_{\text {raw D }}^{j}}{\varepsilon_{\text {prompt D }}^{j}}\right) /\left(\frac{1}{N_{\text {MB trigger }} \varepsilon_{\text {trigger }}} \frac{\left\langle N_{\text {raw D }}\right\rangle}{\left\langle\varepsilon_{\text {prompt D }}\right\rangle}\right)
$$

where the index $j$ identifies the multiplicity interval, $N_{\text {raw D }}^{j}$ is the raw yield, $\varepsilon^{j}$ represents the reconstruction and selection efficiencies for prompt D mesons, $N_{\text {events }}^{j}$ and $N_{\mathrm{MB}}$ trigger are the number of events analysed in each multiplicity interval and in the minimum-bias sample respectively, $\varepsilon_{\text {trigger }}$ is the trigger efficiency. Multiplicity intervals at mid-rapidity are defined by measuring the number of tracklets (track segments joining a pair of space points in the two layers of the Silicon Pixel Detector and aligned with the reconstructed primary vertex) in the pseudorapidity interval $|\eta|<1$. The number of tracklets is converted to the charged-particle multiplicity at mid-rapidity $\left(\mathrm{d} N_{\mathrm{ch}} / \mathrm{d} \eta\right)$ by means of a Monte Carlo simulation. $\mathrm{D}^{0}, \mathrm{D}^{+}$and $\mathrm{D}^{*+}$ self-normalised yields are compatible with each other within the uncertainties in the analyzed multiplicity and $p_{\mathrm{T}}$ intervals. The average $\mathrm{D}^{0}, \mathrm{D}^{+}$and $\mathrm{D}^{*+}$ self-normalised yields as a function of the charged-particle multiplicity at mid-rapidity in different $p_{\mathrm{T}}$ intervals are shown in Fig. 2 (left). The D-meson self-normalised yields increase with the charged-particle multiplicity by a factor of 15 in the range between 0.5 and 6 times $\left\langle\mathrm{d} N_{\mathrm{ch}} / \mathrm{d} \eta\right\rangle$. The enhancement is independent of the transverse momentum within the uncertainties. The mesurement can also be done as a function of the charged-particle multiplicity at forward rapidity, as measured with the signal amplitude of the V0 detector (covering the pseudorapidity region $-3.7<\eta<-1.7$ and $2.8<\eta<5.1$ ) reported in units of the minimumionising-particle charge. In this way, it is possible to introduce a rapidity gap between the region where the D mesons are reconstructed and the region in which the charged-particle multiplicity is measured. An increasing trend is observed also in this case (Fig. 2, right), demonstrating that the trend is not connected to a possible bias due to the pseudorapidity region of the multiplicity mea- 

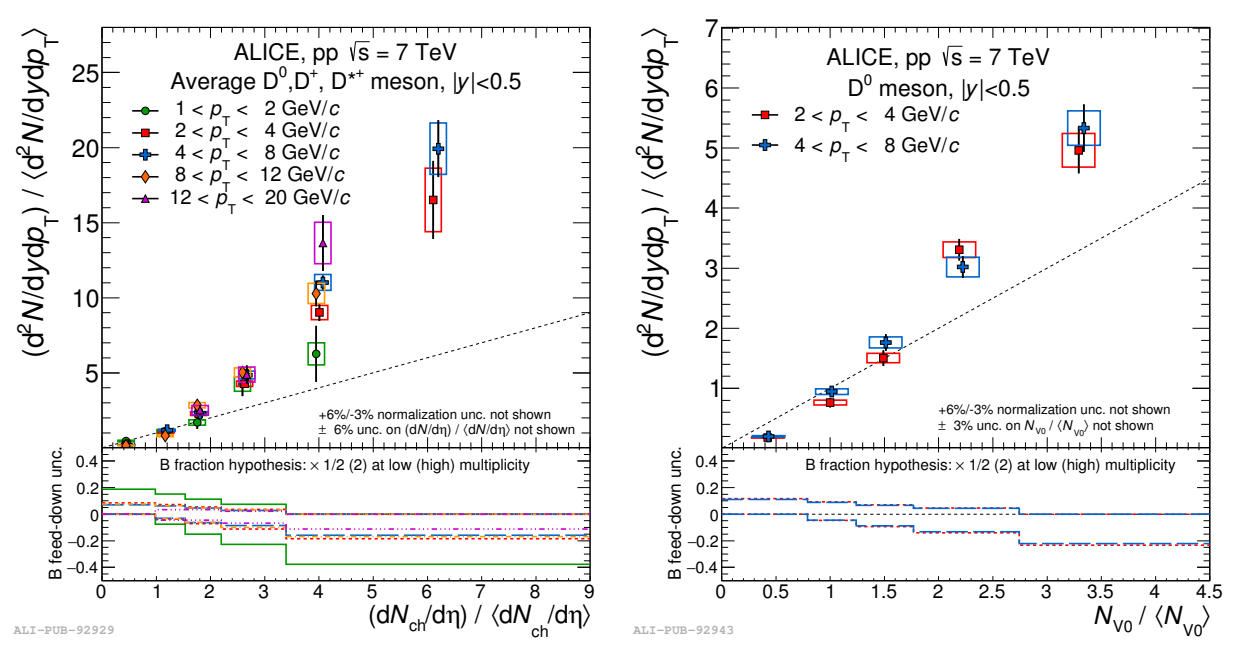

Figure 2: Average $\mathrm{D}^{0}, \mathrm{D}^{+}$and $\mathrm{D}^{*+}$ self-normalised yields as a function of the charged-particle multiplicity at mid-rapidity in different $p_{\mathrm{T}}$ intervals (left). $\mathrm{D}^{0}$ self-normalised yields as a function of the charged-particle multiplicity at forward rapidity in different $p_{\mathrm{T}}$ intervals (right) [8]. Bottom panels show the relative uncertainties due to B feed-down subtraction.
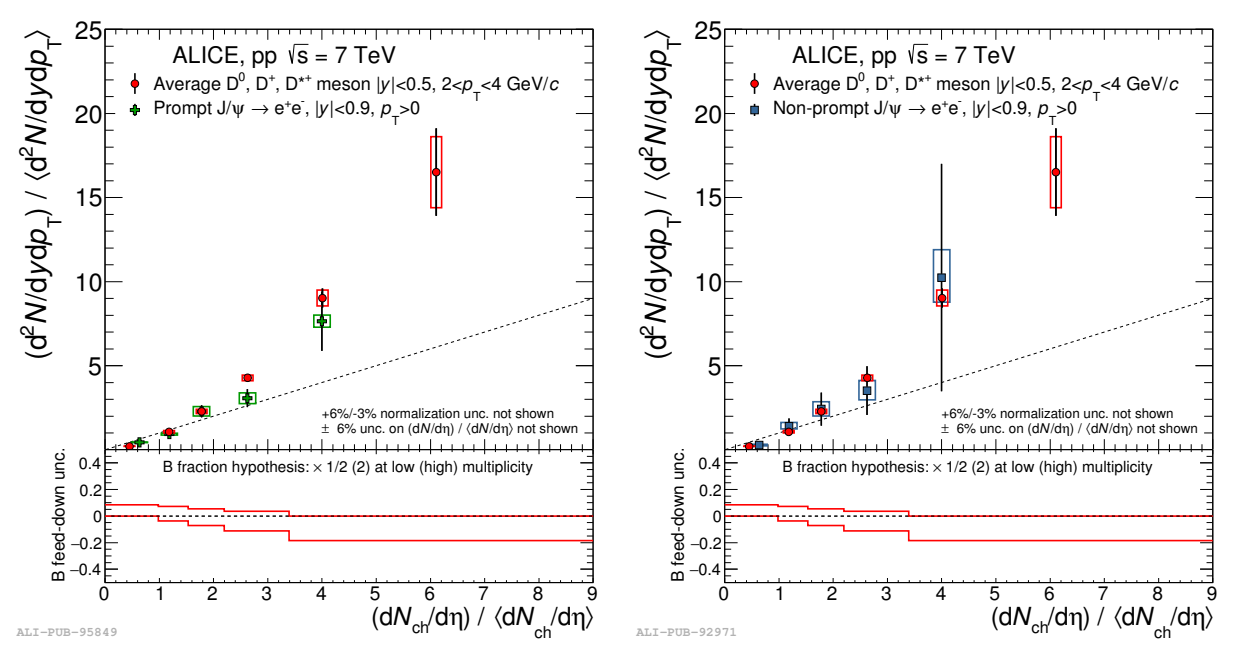

Figure 3: Average D mesons self-normalised yields as a function of the charged-particle multiplicity at mid-rapidity compared with the self-normalised yields of prompt (left) and of non-prompt $J / \psi$ (right) [8]. Bottom panels show the relative uncertainties due to B feed-down subtraction.

surements. Figure 3 shows the D-meson self-normalised yields compared with prompt (left) and non-prompt (right) $\mathrm{J} / \psi$, i.e. coming from the decay of B hadrons. Open charm, open beauty and hidden charm self-normalised yields present a similar increase with charged-particle multiplicity, suggesting that the increasing trend is most likely due to the $c \bar{c}$ and $b \bar{b}$ production processes and is not significantly influenced by hadronisation. Possible explanations of the observed increasing trend include a larger amount of gluon radiation and the contribution of MPI in collisions where heavy quarks are produced. However, more precise measurements are needed for a conclusive 
discrimination of the possible origins of the effect.

\section{Azimuthal correlations of $D$ mesons and charged hadrons}

The azimuthal correlations are built by calculating the difference in $\Delta \eta$ and $\Delta \phi$ between a reconstructed D meson (trigger particle) and the primary charged particles (associated particles). Corrections for limited detector acceptance, spatial inhomogeneities, trigger and associated particle selection efficiency, as well as secondary track contamination are applied. The contribution due to the azimuthal correlations of D mesons from beauty-hadron decays and charged particles is obtained with Monte Carlo simulations and subtracted. Figure 4 (left) shows the D-hadron azimuthal distribution for $\mathrm{D}$ mesons with $5<p_{\mathrm{T}}<8 \mathrm{GeV} / c$ and charged particles with $p_{\mathrm{T}}>0.5 \mathrm{GeV} / c$. The azimuthal correlation distribution is fitted with a double Gaussian (one for the near-side peak centred at $\Delta \phi=0$ and one for the away-side peak centred at $\Delta \phi=\pi)$ on top of the baseline. The baseline is calculated from the region $\pi / 4<|\Delta \phi|<\pi / 2$, and its variation upon redefinition of this interval is treated as a systematic uncertainty. The near-side peak is sensitive to the properties of the jet containing the $\mathrm{D}$ meson. The near-side associated yield, obtained as the integral of the Gaussian centred at $\Delta \phi=0$, is shown in the middle panel of Fig. 4 as function of the D-meson $p_{\mathrm{T}}$. With the present uncertainties it is not possible to conclude about a dependence of the near-side associated yields on the D-meson $p_{\mathrm{T}}$ when associated particles with a $p_{\mathrm{T}}>0.5 \mathrm{GeV} / c$ are considered. The azimuthal correlations of D mesons and charged particles obtained in pp collisions at $\sqrt{s}=7 \mathrm{TeV}$ are compared with PYTHIA 6 [19] and PYTHIA 8 [20] simulations at the same energy using different tunes in the right panel of Fig. 4. The azimuthal correlations from both data and simulations are reported after the subtraction of the baseline. An overall compatibility within the uncertainties is found between data and simulations independently of the considered tunes.
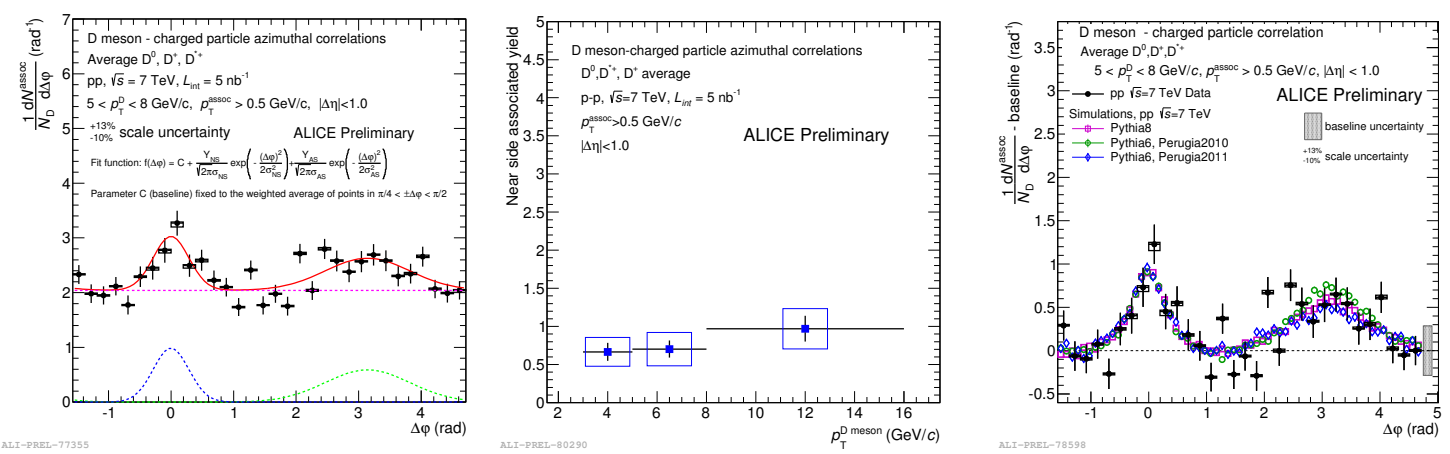

Figure 4: Azimuthal correlations of D mesons and charged particles fitted with an example of fit function (left). Near-side associated yield as a function of D-meson $p_{\mathrm{T}}$ (middle). Comparison of the azimuthal correlations from data with PYTHIA $[19,20]$ simulations (right).

\section{Conclusions}

In summary, the $p_{\mathrm{T}}$-dependent cross sections of prompt $\mathrm{D}$ mesons, leptons from heavy-flavour hadron decays and non-prompt $\mathrm{J} / \psi$ measured with ALICE in pp collisions at $\sqrt{s}=7 \mathrm{TeV}$ and 
$\sqrt{s}=2.76 \mathrm{TeV}$ are well described by different pQCD calculations. The D-meson self-normalized yields exhibit an increasing trend with increasing charged-particle multiplicity in pp collisions, independently of the pseudorapidity region of the multiplicity measurement. The increasing trend can be attributed to the contribution of MPI or to an increased amount of gluon radiation in events with charm. The azimuthal correlations between D mesons and primary charged particles were reported. The azimuthal correlation distributions are consistent with PYTHIA simulations within uncertainties. The analysis of the data from the LHC Run 2 will allow us to explore a higher collision energy and increase the available statistics, enabling more precise measurements in a wider $p_{\mathrm{T}}$ range. The large expected statistics will allow more quantitative and constraining comparisons with expectations from Monte Carlo event generators.

\section{References}

[1] B. A. Kniehl, G. Kramer, I. Schienbein and H. Spiesberger, Phys. Rev. Lett. 96 (2006) 012001

[2] M. Cacciari, M. Greco and P. Nason, JHEP 9805 (1998) 007

[3] R. Maciula and A. Szczurek, Phys. Rev. D 87 (2013) 9, 094022

[4] S. Chatrchyan et al. [CMS Collaboration], Eur. Phys. J. C 73 (2013) 12, 2674

[5] B. Abelev et al. [ALICE Collaboration], JHEP 1309 (2013) 049

[6] M. Aguilar-Benitez et al. [LEBC-EHS Collaboration], Z. Phys. C 41 (1988) 191

[7] B. Abelev et al. [ALICE Collaboration], Phys. Lett. B 712 (2012) 165

[8] J. Adam et al. [ALICE Collaboration], JHEP 1509 (2015) 148

[9] R. Aaij et al. [LHCb Collaboration], JHEP 1206 (2012) 141 [JHEP 1403 (2014) 108]

[10] R. Aaij et al. [LHCb Collaboration], Phys. Lett. B 707 (2013) 52

[11] V. Khachatryan et al. [CMS Collaboration], JHEP 1409 (2014) 094

[12] V. M. Abazov et al. [D0 Collaboration], Phys. Rev. D 90 (2014) 11, 111101

[13] B. Abelev et al. [ALICE Collaboration], JHEP 1201 (2012) 128

[14] B. Abelev et al. [ALICE Collaboration], Phys. Lett. B 718 (2012) 279

[15] B. Abelev et al. [ALICE Collaboration], Phys. Lett. B 721 (2013) 13

[16] B. Abelev et al. [ALICE Collaboration], Phys. Lett. B 708 (2013) 265

[17] B. Abelev et al. [ALICE Collaboration], JHEP 1211 (2012) 065

[18] B. B. Abelev et al. [ALICE Collaboration], Phys. Lett. B 738 (2014) 97 B. Abelev et al. [ALICE Collaboration], JHEP 1207 (2012) 191 B. B. Abelev et al. [ALICE Collaboration], Phys. Rev. D 91 (2015) 1, 012001 B. Abelev et al. [ALICE Collaboration], Phys. Rev. Lett. 109 (2012) 112301

[19] T. Sjostrand, S. Mrenna and P. Z. Skands, JHEP 0605 (2006) 026

[20] T. Sjostrand, S. Mrenna and P. Z. Skands, Comput. Phys. Commun. 178 (2008) 852 